\title{
CERN - EUROPEAN ORGANIZATION FOR NUCLEAR RESEARCH
}

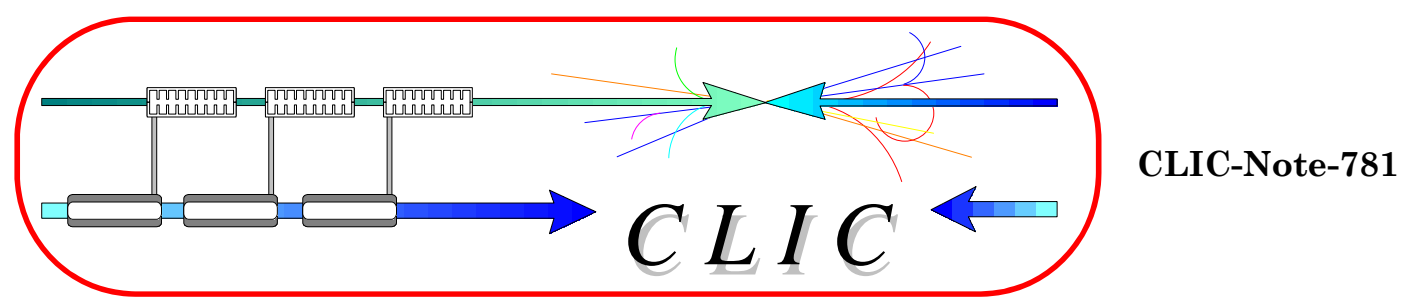

\section{Breakdown Experiments}

S. Calatroni, A. Descoeudres, Y. Levinsen, M. Taborelli, and W. Wuensch

CERN, Geneva, Switzerland

\begin{abstract}
In the context of the CLIC (Compact Linear Collider) project investigations of DC breakdown in ultra high vacuum are carried out in parallel with high power RF tests. From the point of view of saturation breakdown field the best material tested so far is stainless steel, followed by titanium. Copper shows a four times weaker breakdown field than stainless steel. The results indicate clearly that the breakdown events are initiated by field emission current and that the breakdown field is limited by the cathode. In analogy to RF, the breakdown probability has been measured in DC and the data show similar behaviour as a function of electric field. (C2009 American Institute of Physics
\end{abstract}

$13^{\text {th }}$ Advanced Accelerator Concepts 2008

Geneva, Switzerland $22 / 01 / 2009$ 


\title{
DC Breakdown Experiments
}

\author{
S.Calatroni, A.Descoeudres, Y.Levinsen, M.Taborelli, W.Wuensch \\ CERN, European Organization for Nuclear Research, Geneva, Switzerland
}

\begin{abstract}
In the context of the CLIC (Compact LInear Collider) project investigations of DC breakdown in ultra high vacuum are carried out in parallel with high power RF tests. From the point of view of saturation breakdown field the best material tested so far is stainless steel, followed by titanium. Copper shows a four times weaker breakdown field than stainless steel. The results indicate clearly that the breakdown events are initiated by field emission current and that the breakdown field is limited by the cathode. In analogy to RF, the breakdown probability has been measured in DC and the data show similar behaviour as a function of electric field.
\end{abstract}

\section{INTRODUCTION}

An accelerating field of at least $100 \mathrm{MV} / \mathrm{m}$ is necessary in order to build linear colliders in the multi-TeV energy range at acceptable machine length and cost. In the RF (Radio-Frequency) powered accelerating structures the surface electric field will reach even higher values. In the context of the CLIC (Compact Linear Collider) project [1] experimental investigations of the breakdown resistance of materials are underway. In parallel with the high power RF testing, DC measurements are performed on a dedicated setup requiring a simpler infrastructure. The flexibility of the system in terms of sample geometry enables to measure many materials submitted to various treatments. Comparing different materials should enable to distinguish the relevant physical quantities governing breakdown and the experimental dataset can be exploited for verification of breakdown simulations and models including material properties. Measurements of breakdown field and breakdown rate (BDR) are reported in the following sections for various materials.

\section{EXPERIMENTAL SETUP AND MATERIALS}

The setup to investigate DC breakdown has been described previously in detail [2]. An Ultra High Vacuum (UHV) chamber (residual pressure below $10^{-9}$ mbar) hosts the electrodes consisting of a plane grounded cathode (sample) and a hemispherical tip anode of $2.3 \mathrm{~mm}$ diameter. They are separated by a gap of typically $20 \mu \mathrm{m}$, which is

CP1086, Advanced Accelerator Concepts: $13^{\text {th }}$ Workshop, edited by C. B. Schroeder, W. Leemans, and E. Esarey C 2009 American Institute of Physics 978-0-7354-0617-9/09/\$25.00 
adjusted by a mechanical feedthrough having a reproducibility of $+/-1 \mu \mathrm{m}$. The high voltage of up to $+12 \mathrm{kV}$ is provided by a power supply which charges a capacitor of $28 \mathrm{nF}$. In the measurements of breakdown field, the charged capacitor is connected during $2 \mathrm{~s}$ to the anode by using high voltage electromechanical switches. Then the charge on the capacitor is measured and compared to the initial one in order to detect breakdown. If no breakdown occurs the charge on the capacitor is increased further and the cycle is repeated. The breakdown field is calculated assuming parallel plane electrodes, $E_{b}=V / d, V$ being the voltage and $d$ the gap spacing. In breakdown-rate mode the discharge is detected by a $500 \mathrm{MHz}$ current transformer connected to a scope. Direct field-emission current measurements can be made by applying the voltage of the power supply to the anode through a $50 \mathrm{MOhm}$ resistor and measuring the resulting current with a sensitive Ampere meter. Field enhancement values $\beta$ can be calculated from the Fowler-Nordheim formula $[3,4]$ with the assumption that a single tip is dominating the field emission process.

Both electrodes are made of the same material, unless explicitly mentioned. The hemispherical anode is obtained by turning and the plane cathode is a rolled sheet of 1-2 mm thickness. All the metals used in the study are polycrystalline with purities above $99.9 \%$, copper is OFE-copper UNS C10100, stainless steel is the AISI type $316 \mathrm{LN}$ and carbon is graphite. Before insertion in the vacuum chamber the materials were cleaned by the standard procedures used for UHV components at CERN based on detergents or solvents. All the tests were performed after baking of the UHV system including the electrodes at $150 \mathrm{C}$.

\section{RESULTS AND DISCUSSION}

\section{Breakdown resistance of various metals}

A typical conditioning curve up to the maximum breakdown field for molybdenum is shown in figure 1a. After some 50 breakdown events $E_{b}$ reaches a saturation value. A summary of average saturation breakdown fields measured for the various metals is reported in figure $1 \mathrm{~b}$. For each metal at least 3 different spots were measured.

The highest resistance to breakdown is obtained for stainless steel $(830 \mathrm{MV} / \mathrm{m}$ average), followed by titanium. However, for the latter the limiting effect is a strong erosion and material displacement, so that the gap spacing must be continuously readjusted. Copper has a comparatively low $E_{b}(170 \mathrm{MV} / \mathrm{m})$ and has the peculiar property of conditioning in very few sparks. It should be noted that the conditioning speed can change with surface quality or thermal treatment. For molybdenum and copper alloys for instance surface machining by Electro Discharge Machining (EDM) [5] can prolong the conditioning phase by several hundred of sparks and for molybdenum thermal treatments in UHV can reduce the conditioning [6] phase. In all the experiments performed so far on copper and molybdenum with the present setup the $E_{b}$ at saturation did not show sensitivity to the surface and thermal treatments. 
(a)

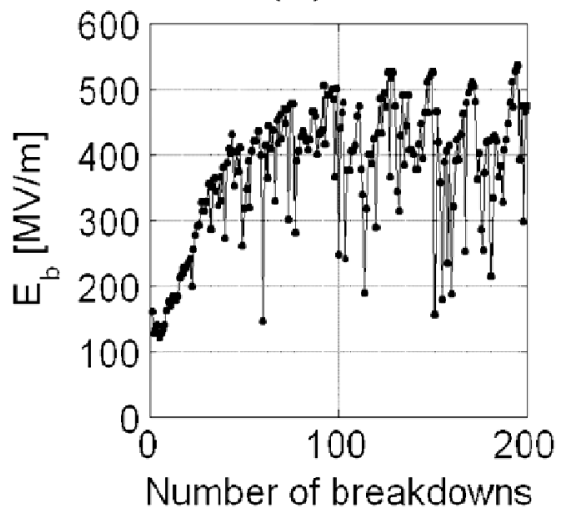

(b)

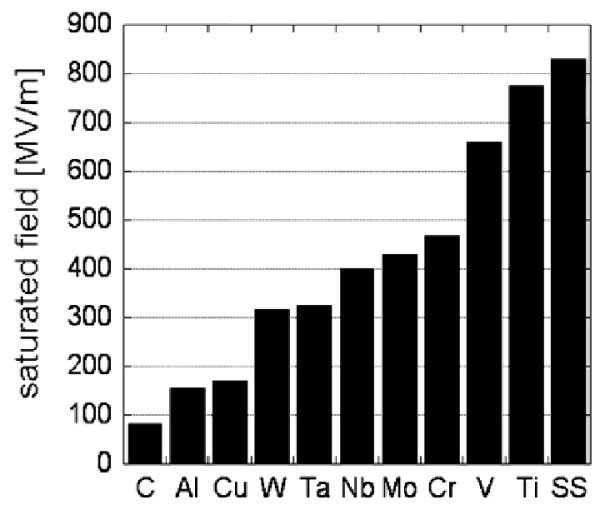

FIGURE 1. (a) Conditioning curve for rolled Mo. (b) Saturated average breakdown field $E_{b}$ obtained for various materials after conditioning.

In order to distinguish whether the breakdown field is limited by the anode or the cathode experiments were performed with electrodes of different materials facing each other. As it is visible from figure 2 the breakdown field in the configuration with a tungsten cathode and titanium anode corresponds to the value found for a system with both electrodes of tungsten (figure 1b). For the configuration having a titanium cathode and a tungsten anode the measured breakdown field is that of titanium. This proves that the resistance to breakdown is limited by the cathode. The fact that in the present geometry with a micrometer size gap the $E_{b}$ is limited by the cathode can be explained by the low energy deposited in the anode. At equal electric field a smaller gap needs a lower voltage and field emitted electrons will deposit only a moderate amount of energy on the anode. The heating effect at the cathode, driven by field emission, will dominate.
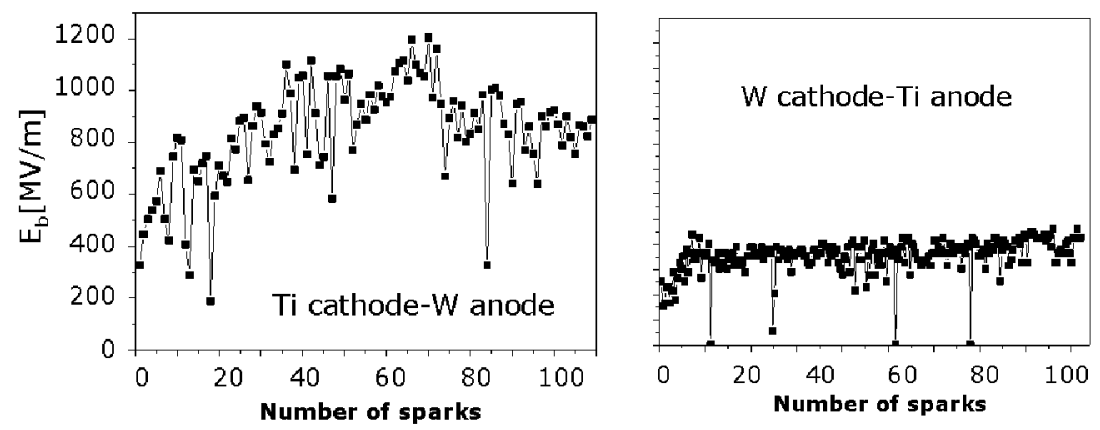

FIGURE 2. Conditioning curves for titanium Ti cathode and $\mathrm{W}$ anode (tip) and for $\mathrm{W}$ cathode and $\mathrm{Ti}$ anode (tip). (same vertical scale).

Field emission from the cathode is considered as a precursor to breakdown $[3,4,7]$. Measurements of cathode current were performed in the present setup for fields well 
below the threshold of breakdown and display field dependence corresponding to the Fowler-Nordheim law. Some values of the calculated field enhancement factor $\beta$ are reported in table 1 .

The $E_{b}$ values given in figure $1 \mathrm{~b}$ are an average over the values measured after the conditioning ramp, where saturation occurs. In the saturation region (figure la) each point corresponds to a spark event, during which the surface can be modified, leading either to improved or worse resistance to breakdown. This is directly visible from the scattering in the values of $E_{b}$ in the saturation region. In this sense the ultimate breakdown field of the material in its best condition would be the highest value reached in the saturation region.

It is clear from the ranking shown in figure $1 \mathrm{~b}$ that many physical quantities are involved in the breakdown process. The breakdown resistance of some of the materials included in this study was measured by Kranjec et al. [8] in crossed cylinders electrode geometry together with the resulting $\beta$ values. In agreement with Alpert [4] their conclusion is that breakdown occurs at a threshold value for the local field $\beta E_{b}$, which is independent of the distance, in contrast to the applied breakdown field $E_{b}$. For some of the materials listed in figure $1 \mathrm{~b}$ the $\beta$ and $\beta E_{b}$ values are compared in table 1. The order of magnitude of the resulting local field $\beta E_{b}$ is the same as in reference 8 , but the ranking of materials with respect to $\beta E_{b}$ is different. Moreover, the reported $\beta$ values at a gap spacing of $25 \mu \mathrm{m}$, which is close to the one used in the present study, are much higher and hence the corresponding $E_{b}$ is much lower. The difference in $\beta$ could be explained by a different conditioning state. Well in line with the value in figure $1 \mathrm{~b}, E_{b}$ of $150 \mathrm{MV} / \mathrm{m}$ for copper has been reported [9] for macroscopic copper electrodes and distances. Furthermore an improvement up to $250 \mathrm{MV} / \mathrm{m}$ upon thermal treatment and polishing was found [9]. In RF experiments with accelerating structures at $30 \mathrm{GHz}[10]$ the ranking for the resistance to breakdown of copper, molybdenum and tungsten is the same as in figure $1 \mathrm{~b}$, if one considers the high breakdown rate data for RF. Copper-stainless steel bi-metallic RF devices have been built following the rationale that stainless steel has larger resistance to breakdown [11].

TABLE 1. Values of the local field and $\beta$ measured in this work in comparison with the data of reference 8 .

\begin{tabular}{|c|c|c|c|c|}
\hline Metal & $\bar{\beta}$ & $\begin{array}{c}\beta \text { for } 25 \\
\text { microns gap } \\
\text { (ref. 8) }\end{array}$ & $\begin{array}{c}\text { Local field } \beta E \\
{[G V / m]}\end{array}$ & $\begin{array}{c}\text { Local field } \\
\text { BE [GV/m] } \\
\text { (ref. 8) }\end{array}$ \\
\hline OFE Copper & 46 & 122 & $7.8+/-1.3$ & $10.1+/-0.5$ \\
\hline Mo & 16 & 63 & $6.9+/-1.9$ & $5.6+/-0.6$ \\
\hline $\begin{array}{l}\text { Stainless steel } 316 \mathrm{LN} \text { (steel } \\
\text { type not specified in ref } 8 \text { ) }\end{array}$ & 6 & 104 & $5.0+/-1.4$ & $8.1+/-0.4$ \\
\hline
\end{tabular}

In the present experiment the energy available for the discharge is around $1 \mathrm{~J}$ and is chosen to be of the same order as in the RF experiments at $30 \mathrm{GHz}$ conducted at CERN. The conditioning process will change as a function of the energy at disposal for each breakdown. For instance in the case of molybdenum conditioning requires removal of the surface oxide [6] and a minimum amount of energy will be necessary 
in order to have an effective conditioning process. Trials with different capacitors to study such an effect are planned.

\section{Breakdown probability}

The RF experiments have shown that for practical applications the information about the breakdown field is only meaningful if the corresponding BDR is also measured. The results for the DC measurements of BDR or breakdown probability are shown in figure $3 \mathrm{a}$ for copper and molybdenum; both samples were previously conditioned. As for the RF case the threshold for breakdown is not sharp but associated with a probability. The instrument enables to reach the $10^{-3}$ probability range and lower values imply unpractical measuring times. As for the RF case a power fit of the type BDR $\sim \mathrm{E}^{\gamma}$, which gives a meaningful BDR of zero at zero field, can be applied. The values of $\gamma$ range from 10-15 and 30-35 for copper and molybdenum, respectively. In RF the data at $30 \mathrm{GHz}$ (fig. 3a) [12] for the BDR as a function of surface electric field, show that the slope $\gamma$ is higher for copper (30) than for molybdenum (20). The slopes can be better appreciated in figure $3 \mathrm{~b}$, where the field is normalized with the value giving a BDR of 1 .

(a)

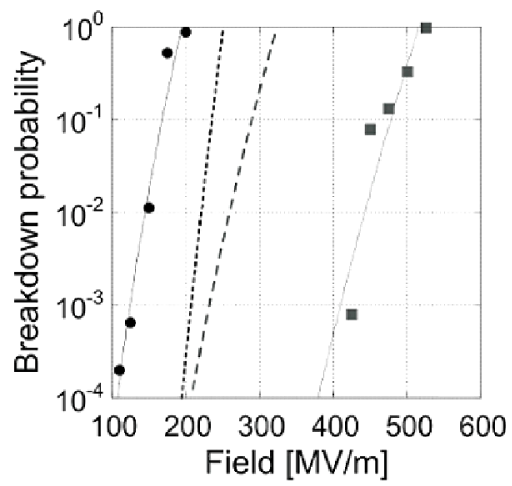

(b)

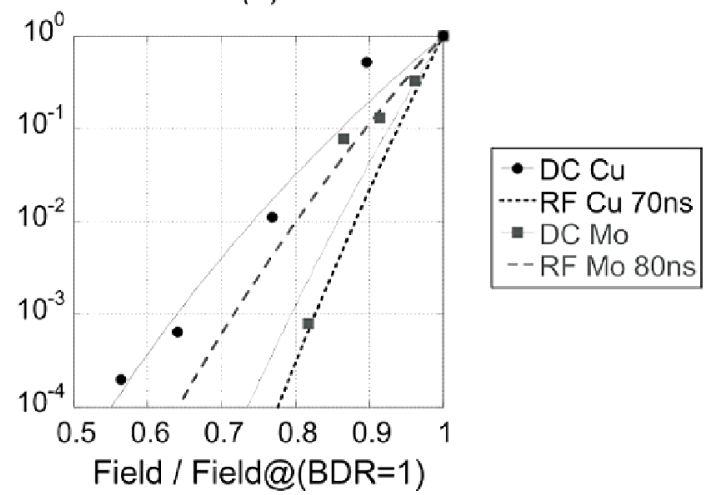

FIGURE 3. (a) Breakdown rate for copper (circles) and molybdenum (squares). Lines indicate the copper (dotted) and molybdenum (dashed) BDR from $30 \mathrm{GHz}$ RF experiments. (b) Same data as a function of the normalized field for BDR $=1$

The field at BDR just below 1 is higher than the average saturation field reported in figure $1 b$, since the surface is not systematically modified or potentially damaged at each attempt. In RF the BDR is known to depend on the pulse length and we arbitrarily chose the data for 70-80 ns. In the present DC experiment the pulse length is not defined precisely. Indeed the charged capacitor is connected to the junction during $1 \mathrm{~s}$, but the effective time of application of the maximum voltage depends on field emission current which can deplete the capacitor charge. With the present setup a constant field emission current of $10 \mathrm{~mA}$ would decrease the charge and hence the BDR by one order of magnitude in 4 milliseconds in copper. The time necessary to get a breakdown event is, however, much shorter and the loss of charges on the capacitor can be neglected. Measurements of the time delay occurring between the voltage ramp 
and the current rise due to breakdown show that most of the events occur in the $100 \mathrm{~ns}$ time scale [13] and some at $1 \mathrm{~ms}$ scale. The fact that two different time domains are found indicates the presence of two distinct mechanisms leading to breakdown.

\section{CONCLUSIONS}

The DC measurements are a simple method of screening for the selection of materials for high field applications. For the dataset existing so far the ranking of the materials corresponds to that found in RF at least in the high breakdown rate regime. Stainless steel is the material giving the highest breakdown field. The ranking of the various materials with very different physical properties indicates that a comprehensive model of breakdown must include many physical quantities. Moreover, the values of the breakdown fields $E_{b}$ could depend on the geometry of the system and experiments at variable distance are in progress in order to verify this aspect, as well as the energy dependence of the conditioning process. The results demonstrate that breakdown rate measurements can be performed also in DC and show a similar general behavior as in RF.

\section{REFERENCES}

1. http://clic-study.web.cern.ch/CLIC-Study/

2. .M. Kildemo, Nucl. Instrum. Methods Phys. Res. A 530, 596 (2004)

3. P.A. Chatterton, Vacuum breakdown, in: J. M. Meek, J. D. Craggs (Eds.), Electrical Breakdown in Gases, Wiley, NewYork, 1978

4. D.Alpert, D.Lee, E.M.Lyman and H.E.Tomaschke, J.Vac.Sci Technol. 1, 35 (1966)

5. A.Descoeudres, CLIC One day workshop on breakdown phenomena, 20 May 2008, CERN Geneva, Switzerland, http://indico.cern.ch/conferenceDisplay.py?confId $=33140$

6. T. Ramsvik, S. Calatroni, A. Reginelli and M. Taborelli, Phys.Rev. ST Accel. Beams 10042001 (2007).

7. R. V. Latham (ed.), High Voltage Vacuum Insulation (Academic Press, 1995), New York

8. P.Kranjec, L.Ruby, J.Vac.Sci.Technol. 4, 94 (1967)

9. S. Kobayashi, .Y Hashimoto, M. Maeyama, Y. Saito and Y. Nagai, Vacuum 47745 (1996)

10. C. Achard, H. Braun, S. Dobert, I. Syratchev, M.Taborelli, I. Wilson, and W. Wuensch, Proceedings of PAC2003 p. 495, Portland, OR, 2003

11. J. Haimson and B. Mecklenburg Proceedings of PAC07, Albuquerque, New Mexico, USA, p 2532

12.W.Wuensch, CLIC-NOTE-706, 2007, CERN, Geneva, Switzerland

13. A.Descoeudres, S.Calatroni, M.Taborelli, Proceedings of EPAC 08, Genova, Italy 